\title{
Anti-Blocking Dynamic Adjustment of Communication Data Transmission based on Blockchain Technology
}

\author{
Fei Gao ${ }^{\mathrm{a}}$, Zhiqiang $\mathrm{Wu}^{\mathrm{a}, \mathrm{b}, *}$, Lei Zhang ${ }^{\mathrm{a}}$, and Zedong $\mathrm{Li}^{\mathrm{a}}$ \\ ${ }^{a}$ School of Information Science and Technology, Tibet University, Lasa, 850000, China \\ ${ }^{b}$ Department of Electrical Engineering, Wright State University, Dayton, $\mathrm{OH} 45435$, USA
}

\begin{abstract}
In order to improve the quality of network communication, it is necessary to transfer anti-blocking dynamic regulation of network communication data. A block chain wireless network data transmission anti-blocking dynamic regulation method based on block chain load equalization regulation technology is proposed. The block chain wireless communication network data transmission anti-blocking dynamic regulation transmission channel model is constructed, the anti-jamming design of block chain wireless communication is carried out by an adaptive multipath interference suppression algorithm, and the data transmission anti-blocking dynamic regulation state characteristic quantity of block chain wireless communication network data is extracted. The data allocation model of block chain wireless communication network is constructed, and the data allocation iteration model of block chain wireless communication network is obtained according to the different characteristics of data transmission impedance. The anti-blocking dynamic adjustment optimization of block chain wireless communication networks is realized, and the anti-jamming and security of data transmission anti-blocking dynamic regulation are improved. The simulation results show that the channel balance and output signal-to-noise ratio (SNR) of block-chain wireless communication networks are better, the anti-jamming ability is enhanced, the anti-blocking dynamic adjustment ability of communication data transmission is improved, the output bit error rate is reduced, and the security and load ability of data communication are increased.
\end{abstract}

Keywords: block chain technology; communication data; transmission; anti-blocking; dynamic regulation

(Submitted on October 28, 2019; Revised on November 30, 2019; Accepted on December 16, 2019)

(C) 2019 Totem Publisher, Inc. All rights reserved.

\section{Introduction}

With the development of network communication technology, higher requirements are put forward for the security and anti-interference of the network [1-3]. It is necessary to carry out anti-blocking dynamic adjustment control of block chain wireless communication network data transmission. In reference [4], a data equalization method of block chain wireless communication network based on direct sequence spread spectrum was proposed. The autocorrelation matching filter was designed, and the data anti-blocking dynamic regulation algorithm optimization and channel equalization design of block chain wireless communication networks were realized by the direct sequence spread spectrum method. However, the channel equalization information of this method was not good, and the adaptive equalization control ability was not strong. A channel equalization allocation technique based on envelope detection modulation was proposed in reference [5]. The wavelet basis function was used to detect and filter the input wireless communication network data of communication channels. Combined with the fuzzy clustering method, the block dynamic regulation of block chain wireless communication network data transmission was realized, but the method had poor anti-interference [6].

In order to solve the above problems, an anti-blocking dynamic adjustment method for block chain wireless communication network data transmission based on block chain load balancing and adjustment technology is proposed. 


\section{Data Transmission Channel Model for Block Chain Wireless Communication Networks}

In order to realize the transmission anti-blocking dynamic adjustment of block chain wireless communication network data, it is necessary to construct a data transmission channel model of block chain wireless communication networks, combine the channel output structure of block chain wireless communication network data to carry out balanced control, adopt an adaptive random link forwarding control protocol, carry out the balanced control of block chain wireless communication network data transmission, and adopt fractional interval equilibrium control technology [7]. The load of block chain wireless communication network data is $E_{j}=\sum_{k}\left|C_{j}(k)\right|^{2}$. According to the load of anti-blocking dynamic adjustment of block chain wireless communication network data transmission, the fuzzy adaptive equilibrium scheduling method is used to control the transmission anti-blocking dynamic adjustment of the network. The learning function of block chain wireless communication network data transmission is as follows:

$$
\left\{\begin{array}{l}
\min \sum_{1 \leq i \leq K} \sum_{e \subseteq k(e)} \frac{f(e(i))}{C(e, i)} \\
0 \leq f(e, i) \leq C(e, i) \\
F=\text { const } \\
\quad \sum_{1 \leq i \leq K, e \subseteq k(e)} \frac{f(e(i))}{C(e, i)}+\sum_{e \subseteq k(e)} \frac{f\left(e^{\prime}(i)\right)}{C\left(e^{\prime}, i\right)} \leq k(v)
\end{array}\right.
$$

The link random allocation method is used to detect the data output of block chain wireless communication network. The spatial sampling load of block chain wireless communication network data is as follows:

$$
\begin{aligned}
\operatorname{Computition}\left(n_{j}\right) & =\left(E_{\text {elec }}+E_{D F}\right) l \delta+E_{T x\left(l, d_{j}\right)} \\
& =\left(E_{\text {elec }}+E_{D F}\right) l \delta+l E_{\text {elec }}+l \varepsilon_{f s} d_{j}{ }^{2} \\
& =\left[\left(E_{\text {elec }}+E_{D F}\right) \delta+E_{\text {elec }}+\varepsilon_{f s} d_{j}{ }^{2}\right] l
\end{aligned}
$$

The frequency domain correlation characteristic of sensing communication big data is extracted, and the propagation loss of storage medium $O D$ on the $w$ path $\omega$ is analyzed. The load balancing design of block chain wireless communication networking data is carried out by using the link random distribution method, the autocorrelation matching filter is designed, and the multi-path suppression of block chain wireless communication network data transmission is realized by the direct sequence spread spectrum method [8-9]. The output load $\xi_{k}^{w}(\omega)$ balancing scheduling of the network is obtained as follows:

$$
\eta_{k}^{w}(\omega)=E\left(T_{k}^{w} \mid T_{k}^{w}>\xi_{k}^{w}(\omega)\right), \quad k \in R_{w}, w \in W
$$

The adaptive adjustment error $\xi_{k}^{w}(\omega)$ of the anti-interference block chain wireless communication network can be expressed as follows:

$$
\xi_{k}^{w}(\omega)=\min \left\{\xi \mid \operatorname{Pr}\left(T_{k}^{w} \leq \xi\right) \geq \omega\right\}=E\left(T_{k}^{w}\right)+\gamma_{k}^{w}(\omega), \quad k \in R_{w}, w \in W
$$

The statistical characteristics of block chain wireless communication networking data are extracted, and the link overhead is $E\left(T_{k}^{w}-\xi_{k}^{w}(\omega) \mid T_{k}^{w} \geq \xi_{k}^{w}(\omega)\right)$. The block chain wireless communication networking data can be expressed as follows:

$$
s_{h}^{w}=E\left[\min _{k \in R_{w}}\left\{\mathrm{H}_{h, k}^{w}\right\} \mid \eta^{w}\right]=-\frac{1}{\theta} \ln \sum_{k \in R_{w}} \exp \left(-\theta \eta_{h, k}^{w}(\omega)\right), \quad w \in W, h \in H
$$


The impulse response function of anti-interference block chain wireless communication is obtained by introducing a feedback branch. The iterative formula of traffic sequential adaptive prediction is expressed as follows:

$$
\xi_{k}^{w}(\omega)=t_{k}^{w}+\Phi^{-1}(\omega) \sigma_{k, t}^{w}, \quad k \in R_{w}, w \in W
$$

The impulse response characteristic of the network communication is as follows:

$$
\eta_{k}^{w}(\omega)=t_{k}^{w}+\sigma_{k, t}^{w} / \sqrt{2 \pi}(1-\omega) \exp \left(-\left(\Phi^{-1}(\omega)\right)^{2} / 2\right)
$$

The channel model of block chain wireless communication network data transmission blocking dynamic adjustment is constructed. The subsequence of channel transmission anti-blocking dynamic adjustment scheduling set is represented by using a time reversal mirror.

$$
\begin{aligned}
& r_{1}(n)=r_{2}(n) \exp \left(-\mathrm{j} \omega_{0} T_{p} / 2\right), \quad n=0,1, \cdots,(N-3) / 2 \\
& r_{2}(n)=A \exp \left[\mathrm{j}\left(\omega_{0} n T+\theta\right)\right], \quad n=0,1, \cdots,(N-3) / 2
\end{aligned}
$$

Perform $(N-1) / 2$ point Fourier transform for $r_{1}(n)$ and $r_{2}(n)$ to realize the optimal design of the data transmission channel for block chain wireless communication networks [10-11].

\section{Optimization of Anti-blocking Dynamic Regulation for Data Transmission in Block Chain Wireless Communication Networks}

The data transmission congestion dynamic adjustment model of block chain wireless communication networks is constructed. Combined with the correlation fusion model [12-13], the impulse response of block chain wireless communication network data output is obtained by using a decentralized control protocol under the condition of static mapping.

$$
\left\{\begin{array}{l}
y(t)=x\left(t-t_{0}\right) \Rightarrow W_{y}(t, v)=W_{x}\left(t-t_{0}, v\right) \\
y(t)=x(t) e^{\mathrm{j} 2 \pi v_{0} t} \Rightarrow W_{y}(t, v)=W_{x}\left(t, v-v_{0}\right)
\end{array}\right.
$$

The impulse response of block chain wireless communication network data is calculated. In big data transmission anti-blocking dynamic adjustment of anti-interference block chain wireless communication, link allocation is carried out according to the synthesis characteristics of the anti-interference block chain wireless communication channel [14-15]. The transmission anti-blocking dynamic adjustment normal distribution of block chain wireless communication network data satisfies $X \sim S_{\alpha}(1, \beta, 0)$, where $1<\alpha<2$. The inertia characteristic distribution of anti-blocking dynamic adjustment of block chain wireless communication network data transmission is as follows:

$$
\begin{aligned}
& y(t)=\sqrt{k} x(k t), k>0 \\
& W_{y}(t, v)=W_{x}(k t, v / k)
\end{aligned}
$$

Where $k$ represents the characteristic resolution of blocking dynamic adjustment of block chain wireless communication network data transmission, $v$ represents the modulation frequency, and $W_{x}$ is the joint state estimation of anti-blocking dynamic adjustment load of block chain wireless communication network data transmission [16-17]. The load response control model of anti-blocking dynamic adjustment for block chain wireless communication network data transmission is calculated and expressed as follows: 


$$
\Phi(\omega)=E\left[e^{\mathrm{j} \omega X}\right]=\left\{\begin{array}{l}
\exp \left\{\mathrm{j} \mu \omega-|\sigma \omega|^{\alpha}\left[1-\mathrm{j} \beta \operatorname{sgn}(\omega) \tan \left(\frac{\pi \alpha}{2}\right)\right]\right\}, \alpha \neq 1 \\
\exp \left\{\mathrm{j} \mu \omega-|\sigma \omega|^{\alpha}\left[1+\mathrm{j} \beta \operatorname{sgn}(\omega) \frac{2}{\pi} \ln |\omega|\right]\right\}, \alpha=1
\end{array}\right.
$$

In embedded environments, the $j=0,1, \cdots, M$ sampling point output of anti-blocking dynamic adjustment state characteristic quantity of block chain wireless communication network data transmission is expressed as follows:

$$
x(t) \mathrm{e}^{\mathrm{j} \pi t^{2} \cot \alpha}=\sum_{n=-N}^{N} x\left(\frac{n}{2 \Delta x}\right) \mathrm{e}^{\frac{\mathrm{j} \pi(\cot \alpha) n^{2}}{(2 \Delta x)^{2}}} \sin c\left[2 \Delta x\left(t-\frac{n}{2 \Delta x}\right)\right]
$$

Where $N=(\Delta x)^{2}$ represents the statistical peak value of the output data of block chain wireless communication network data. Combined with the fuzzy matching filter identification method, the output symbol modulation of block chain wireless communication network data is carried out, and the symbol modulation output of block chain wireless communication network data transmission anti-blocking dynamic adjustment is obtained as follows:

$$
m_{k}=\left.\Phi^{k}(\omega)\right|_{\omega=0}=E\left[x^{k}\right]=\int_{-\infty}^{+\infty} x^{k} f(x) \mathrm{d} x
$$

$m_{1}=E(x)=\eta \quad$ uses adaptive frequency separation technology to obtain the decoding characteristics of the network.

$$
m_{k}=E\left[x^{k}\right]= \begin{cases}0, & k \text { is odd } \\ 1 \cdot 3 \cdots(k-1) \sigma^{2}, & k \text { is even }\end{cases}
$$

The fractional interval balancing method is used to control the anti-blocking dynamic adjustment of block chain wireless communication network data transmission, and the optimization of anti-blocking dynamic adjustment of block chain wireless communication network data transmission is realized.

$$
G\left(U \mid \mu_{k}, \sum_{k}\right)=(2 \pi)^{-d / 2}\left|\sum_{k}\right|^{-1 / 2} \times \exp \left[-\frac{1}{2}\left(U-u_{k}\right)^{\mathrm{T}} \sum_{k}^{-1}\left(U-u_{k}\right)\right]
$$

In that above formula, $G\left(U \mid \mu_{k}, \sum_{k}\right)$ is the feature quantity of the anti-blocking dynamic adjustment of the data transmission of the block-chain wireless communication network, $U$ represents the bandwidth of the channel, and $u_{k}$ represents the symbol sequence of the block-chain wireless communication network data transmission anti-blocking dynamic adjustment. In this paper, the anti-blocking dynamic adjustment process of the data transmission of block-chain wireless communication network is realized.

\section{Optimization of Dynamic Adjustment Algorithm for Data Anti-blocking in Communication Wireless Communication Network}

\subsection{Anti-Interference Design of Adaptive Noise Cancellation Filter}

A channel model of block-chain wireless communication network data transmission blocking dynamic adjustment is constructed. The characteristic recombination of a transmission channel in a block-chain wireless communication networking channel is released by adopting block-chain space-balancing control technology and a block-chain wireless communication networking channel. The statistical time sequence of the wireless communication networking data of the input block chain is $x_{n}$, the expected response is $d_{n}$, and the frequency response is as follows:

$$
d(t)=a(t) c(t)=\sum_{n=0}^{\infty} d_{n} g_{c}\left(t-n T_{c}\right)
$$


Wherein

$$
d_{n}=\left\{\begin{array}{ll}
+1 & a_{n}=c_{n} \\
-1 & a_{n} \neq c_{n}
\end{array},(n-1) T_{c} \leq t \leq n T_{c}\right.
$$

According to the above results, there is intersymbol interference in multi-path channels, so adaptive noise cancellation filter is used to suppress interference. The iterative formula of adaptive noise cancellation filter anti-interference is as follows:

$$
\hat{e}(k)=z_{R}(k)\left(\left|z_{R}(k)\right|^{2}-\hat{s}_{R}^{2}(k)\right)+j z_{I}(k)\left(\left|z_{I}(k)\right|^{2}-\hat{s}_{I}^{2}(k)\right)
$$

In the formula, $\operatorname{sgn}(\cdot)$ represents the symbolic function, and $\hat{s}_{I}(k)$ and $\hat{s}_{R}(k)$ are the normalization amplitude and tap interval of the adaptive noise cancellation filter, respectively. In the process of data transmission blocking dynamic adjustment in block chain wireless communication networks [18-19], the ambiguity identification and processing are carried out according to the different impedance characteristics of communication data transmission, and the adaptive adjustment iterative function of data transmission blocking dynamic regulation in block chain wireless communication network is obtained as

$$
\begin{aligned}
\boldsymbol{f}_{F}(k+1)=\boldsymbol{f}_{F}(k)-\mu \cdot \nabla_{\boldsymbol{f}_{F}(k)} J_{\text {MMDMMA }} \\
=\boldsymbol{f}_{F}(k)-\mu \cdot \nabla_{\boldsymbol{f}_{F}(k)}\left(J_{\text {MMDMMA_R }_{-}}+J_{\text {MMDMMA_I }_{-}}\right) \\
=\boldsymbol{f}_{F}(k)-\mu_{F}\left[\rho(k) e_{\text {MDMMA }}(k)+(1-\rho(k)) e(k)\right] \boldsymbol{y}^{*}(k) \\
=\boldsymbol{f}_{F}(k)-\mu_{F}\left[\rho(k)\left(e_{\text {MDMMA_R }_{-}}(k)+j e_{\text {MDMMA_I }_{-}}(k)\right)+\right. \\
\\
\left.(1-\rho(k))\left(e_{R}(k)+j e_{I}(k)\right)\right] \boldsymbol{y}^{*}(k)
\end{aligned}
$$

According to the principle of consistency estimation, the filtering fusion of the data transmission blocking dynamic adjustment of the block chain wireless communication network is realized, and the anti-interference is carried out.

\subsection{Channel Equalization and Optimization of Data Transmission Allocation in Wireless Communication Networks}

According to the filter output of block chain wireless communication networking data, the channel distortion in the received signal is analyzed, and the adaptive allocation of block chain wireless communication networking data is carried out. The results are as follows:

$$
|s(f)|=A \sqrt{\frac{1}{2 k}}\left\{\left[c\left(v_{1}\right)+c\left(v_{2}\right)\right]^{2}+\left[s\left(v_{1}\right)+s\left(v_{2}\right)\right]^{2}\right\}
$$

Where $A(t)$ is the sampling amplitude of block chain wireless communication network data, $f_{0}$ is the initial frequency of block chain wireless communication network data transmission, $k=B / T$ is the frequency modulation slope, and $\mathrm{B}$ is the fractional interval equilibrium coefficient. Combined with the fuzzy $\mathrm{C}$-means clustering method, the spatial structure of block chain wireless communication network data is reorganized, and the discrete scheduling method of sampling normalization is used to obtain the balanced distribution characteristic of the block chain wireless communication network's channel impulse response function.

$$
E=\|x(t)\|^{2}=\sum_{j} \sum_{k}\left|C_{j}(k)\right|^{2}=\sum_{j} E_{j}
$$

Where the characteristic distribution coefficient of modulation and demodulation can be expressed as follows:

$$
g(t)=\frac{1}{\sqrt{a_{0}}} f\left(\frac{t-\tau_{0}}{a_{0}}\right)
$$


Where $t_{0}=f_{0} T / B$ and $K=T f_{i}\left(-\frac{T}{2}\right) f_{i}\left(\frac{T}{2}\right) / B$. The energy compensation of the block chain wireless communication network is carried out, and the block chain wireless communication network data balanced allocation power spectrum density is $P_{j}=E_{j} / E$. The reorganization multi-meridian component expression is

$$
\begin{gathered}
y(n)=x(n) \times h(n)+n(n) \\
\tilde{x}(n)=y(n) \times W(n) \\
\hat{x}(n)=g(\tilde{x}(n))
\end{gathered}
$$

Where $\hat{x}(n)$ is the spatial estimation value of block chain wireless communication network data, $h(n)$ is the block chain wireless communication network channel fading characteristic, $n(n)$ is the interference component, $W(n)$ is the multi-path response characteristic of the block chain wireless communication network, and the optimal detection function of block chain wireless communication network data transmission blocking dynamic adjustment is obtained.

$$
\left\{\begin{array}{l}
H_{0}: x^{\prime}(t)=w(t) \\
H_{1}: \sqrt{E} s^{\prime}(t)+w(t)
\end{array}, \quad 0 \leq t \leq T\right.
$$

Wherein

$$
x^{\prime}(t)=x(t) \times h_{w}(t), \quad s^{\prime}(t)=s(t) \times h_{w}(t)
$$

In summary analysis, the balanced distribution of block chain wireless communication networking data and anti-inter-code interference processing are realized.

\section{Simulation Experiment and Result Analysis}

In order to test the application performance of this method in the realization of block chain wireless communication network data transmission anti-blocking dynamic adjustment, an experimental test is carried out, and the simulation analysis is carried out using MATLAB. It is assumed that the distribution area of block chain wireless communication network data transmission is a uniform square area of $400 \mathrm{~m} \times 400 \mathrm{~m}$, the sample sequence bandwidth of block chain wireless communication network data output is $140 \mathrm{Gbit}$, and the intensity of inter-symbol interference of network communication data transmission is $-10-10 \mathrm{~dB}$. The adaptive symbol transmission rate is $10 \mathrm{kBaud}$. According to the above parameters, the simulation model of anti-blocking dynamic adjustment of data transmission in block chain wireless communication networks is constructed. The sampling time interval of the transmission symbol is $0.12 \mathrm{~s}$, and the output of anti-blocking dynamic adjustment of block chain wireless communication network data transmission is shown in Figure 1.

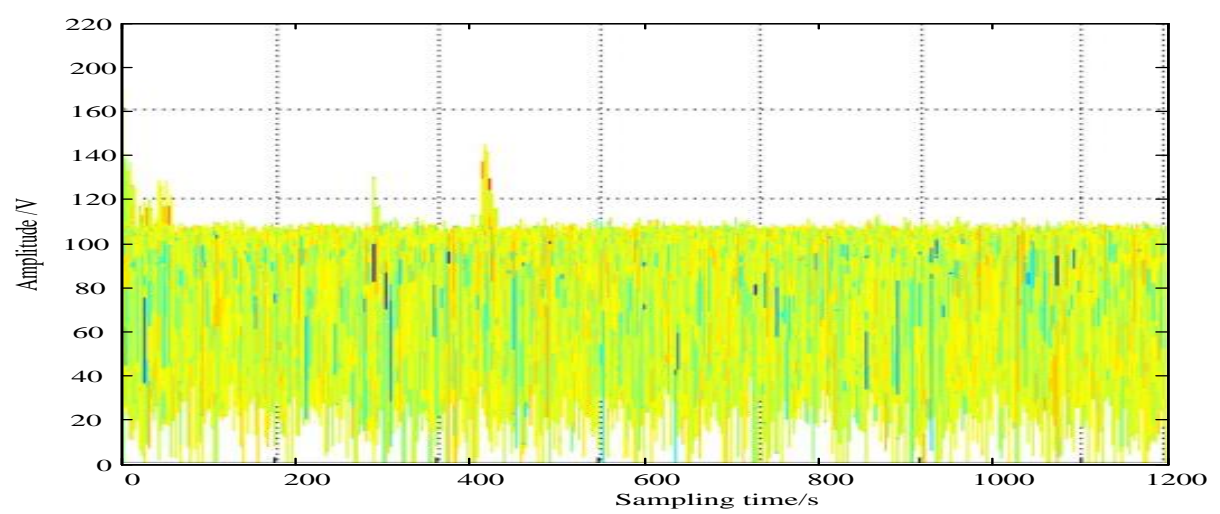

Figure 1. Anti-blocking dynamic regulation of data transmission in block chain wireless communication network 
Taking the data of Figure 1 as the research object, the transmission anti-blocking dynamic adjustment state characteristic of block chain wireless communication network data is extracted, and the fractional interval balancing method is used to control the anti-blocking dynamic adjustment of block chain wireless communication network data transmission. The output is shown in Figure 2.

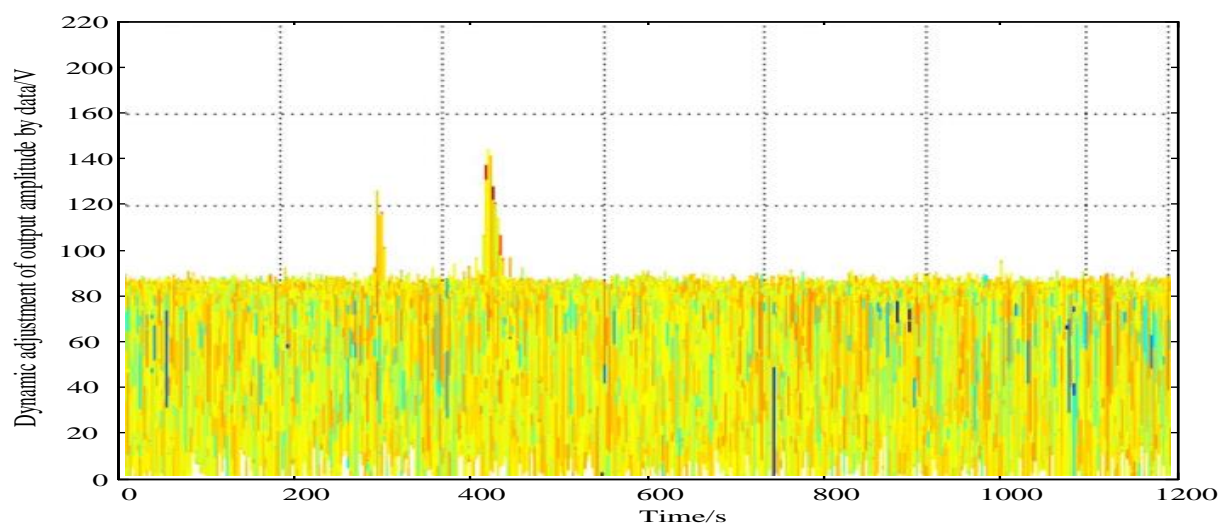

Figure 2. Block chain wireless communication network data transmission anti-blocking dynamic adjustment output

Figure 2 shows that the output balance and noise cancellation performance of block chain wireless communication network data transmission anti-blocking dynamic adjustment are better. Assume that the distribution area of data transmission in block chain wireless communication network is a uniform square area of $200 \mathrm{~m} \times 200 \mathrm{~m}$, and the distribution radius SF of wireless sensor nodes is $10 \mathrm{~m}$. The length of the data sample sequence monitored by the sensor network is 1,024 , the delay of the multi-path spread is $1.2 \mathrm{Ms}$, the envelope replication is $12 \mathrm{~V}$, the multi-path amplitude parameter is (1, 0.5, $0.3,0.15,0.15)$, the multi-path delay parameter is $(0,1.3,2.3,3.6,4.9)$, the symbol rate of the block chain wireless communication network is $1 \mathrm{kBaud}$, and the carrier frequency is $24 \mathrm{kHz}$. According to the above simulation environment and parameters, the simulation analysis of data transmission congestion dynamic adjustment in block chain wireless communication network is carried out, and the time domain waveform of data sampling in wireless communication network is obtained as shown in Figure 3.

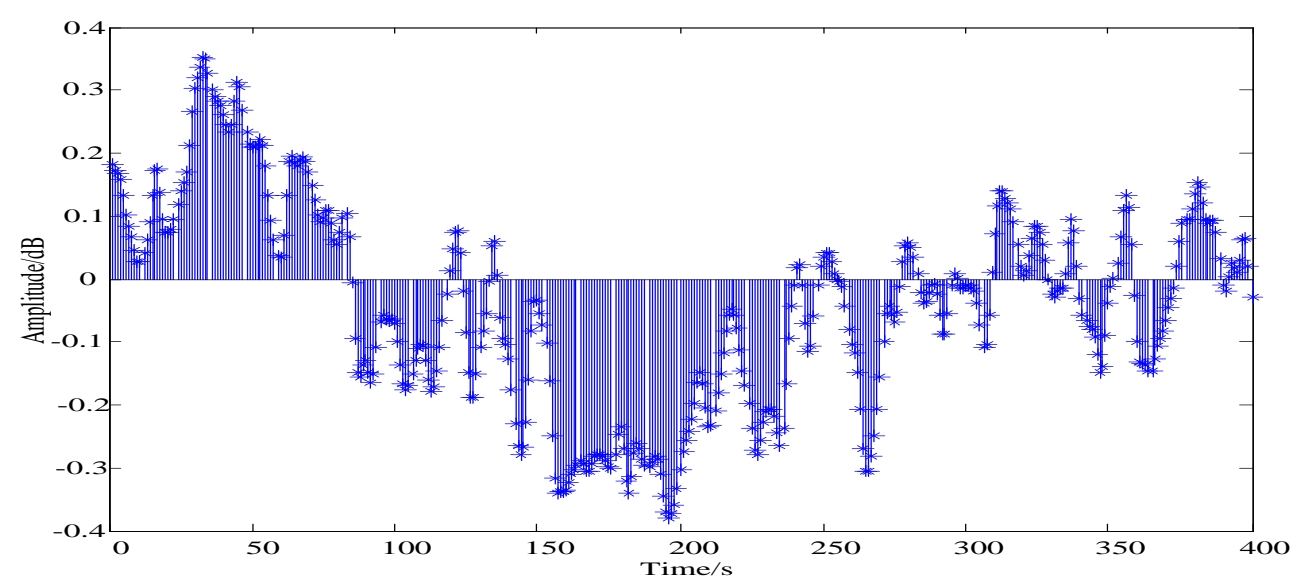

Figure 3. Time domain waveform of data sampling in wireless communication network

The sampling results of wireless communication network data in Figure 3 are allocated by the block chain wireless communication network. The characteristic reorganization of the transmission channel in the block chain wireless communication network channel is realized by using block chain spatial equilibrium control technology, and the anti-interference processing is realized. The anti-interference output of block chain wireless communication network data is shown in Figure 4.

Figure 4 shows that the blocking dynamic adjustment of block chain wireless communication network data is carried out using this method, the anti-interference ability of output is enhanced, the inter-symbol interference data is effectively filtered, the output signal-to-noise ratio is improved to $24 \mathrm{~dB}$, the anti-blocking dynamic adjustment optimization of block chain wireless communication network is realized, and the bit error rate of wireless communication network data balanced allocation output is tested in the 2BPSK, 4BPSK, 8BPSK, and 16 QAM modulation modes. The output bit error rate (BER) 
is analyzed, and the test results are shown in Figure 5.

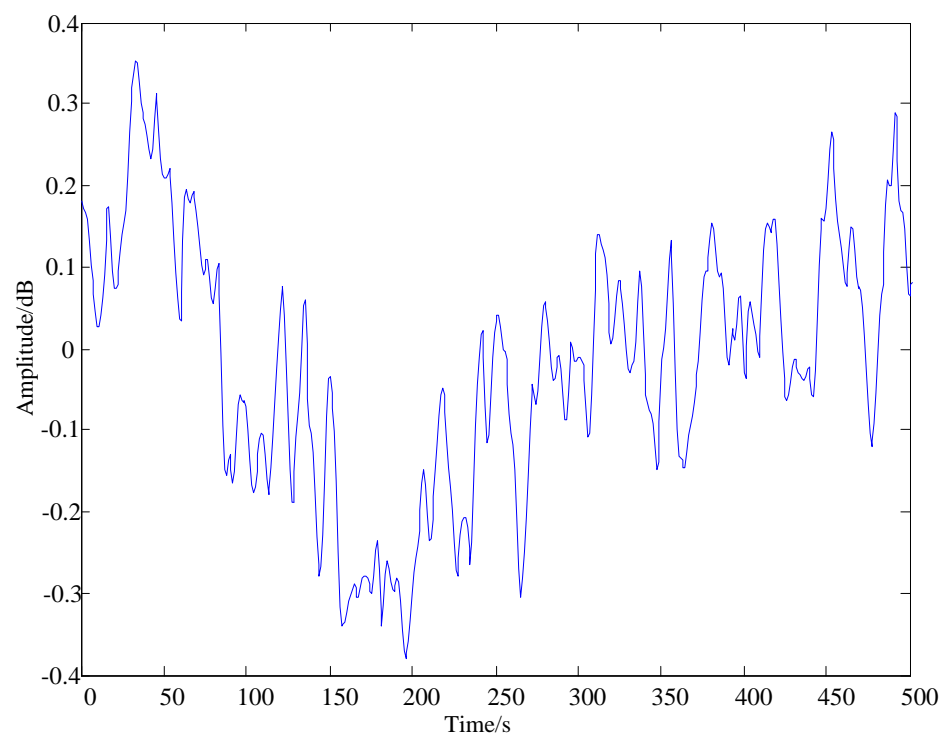

Figure. 4 Anti-interference output of block chain wireless communication network data

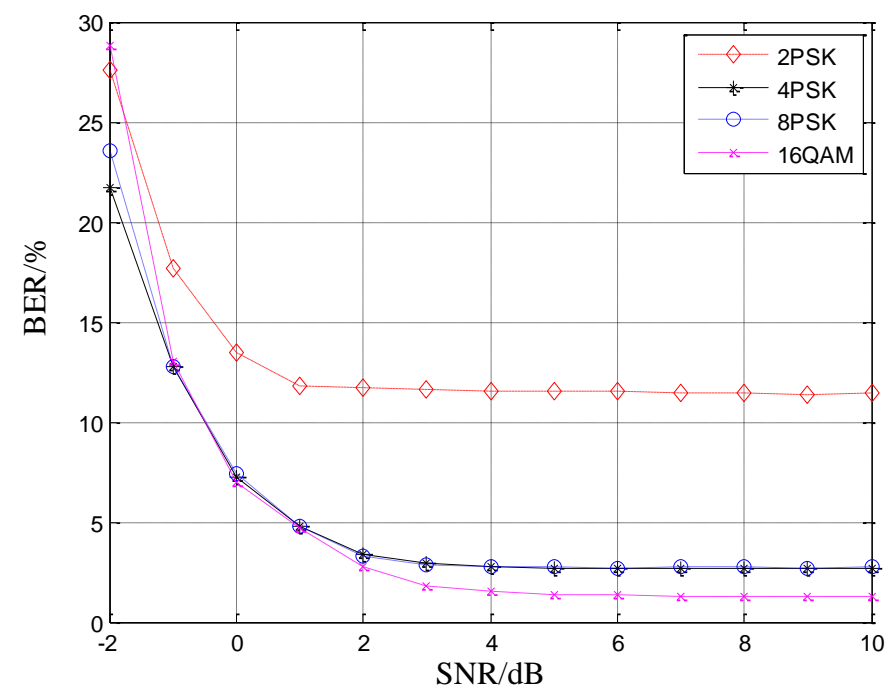

Figure 5. Output bit error rate test

It is found that the data anti-blocking dynamic adjustment algorithm of block chain wireless communication networks is optimized by using this method, and the output bit error rate is low. With an increase in the modulation bandwidth, the bit error rate decreases. When the input signal-to-noise ratio is $4 \mathrm{~dB}$, the output error rate of block chain wireless communication network reaches its lowest and converges to 0.021 , which meets the requirements of communication equilibrium. The output bit error rate (BER) is tested, and the comparison results are shown in Table 1. The analysis shows that the output BER of block chain wireless communication network data transmission anti-blocking dynamic adjustment is low.

Table 1. Comparison of output BER

\begin{tabular}{cccc}
\hline SNR/dB & Proposed method & Reference [3] & Reference [4] \\
\hline-10 & 0.112 & 0.154 & 0.134 \\
0 & 0.075 & 0.095 & 0.114 \\
10 & 0 & 0.032 & 0.068 \\
\hline
\end{tabular}




\section{Conclusions}

In this paper, a block chain wireless communication network data transmission anti-blocking dynamic regulation method based on block chain load equalization adjustment technology was proposed. The link random allocation method was used to filter and detect the data output of block chain wireless communication networks, an autocorrelation matching filter was designed, and multipath suppression of block chain wireless communication network data transmission was realized through the direct sequence spread spectrum method. A channel model of block chain wireless communication network data transmission blocking dynamic regulation was constructed, and a time reversal mirror was adopted. The block chain wireless communication network data transmission anti-blocking dynamic regulation optimization was realized, and the anti-interference and security of data transmission anti-blocking dynamic regulation were realized. It was found that this method can improve the security of block chain wireless communication network data transmission anti-blocking dynamic regulation and reduce the output error rate.

\section{Acknowledgments}

This paper is supported by the 2017 Key R\&D Plan of the Autonomous Region (No. XZ201703-GC-09), 2019 Key Project of the Natural Fund of the Autonomous Region (No. XZ2019ZRG-24), and National Science Foundation (No. 1748494).

\section{References}

1. S. Niu, Q. Chen, D. Sisternes, et al, "Robust Noise Region-based Active Contour Model via Local Similarity Factor for Image Segmentation," Pattern Recognition, Vol. 3, No. 61, pp. 104-119, 2016

2. J. Y. Zhang, H. P. Zhao, and S. Chen, "Face Recognition based on Weighted Local Binary Pattern with Adaptive Threshold," Jounal of Electronics and Information Technology, Vol. 36, No. 6, pp. 1327-1333, 2014

3. I. Naseem, R. Togneri, and M. Bennamoun, "Linear Regression for Face Recognition," IEEE Transactions on Pattern Analysis and Machine Intelligence, Vol. 32, No. 11, pp. 2106-2112, 2010

4. A. Wagner, J. Wright, A. Ganesh, et al, "Toward a Practical Face Recognition System: Robust Alignment and Illumination by Sparse Representation," IEEE Transactions on Pattern Analysis and Machine Intelligence, Vol. 34, No. 2, pp. 372-386, 2012

5. W. Xiang, L. Zhang, Y. H. Chen, et al, "Single Image Super Resolution Combining with Structural Self-Similarity and Convolution Networks," Journal of Computer Applications, Vol. 38, No. 3, pp. 854-858, 2018

6. X. You, W. Xue, J. Lei, et al, "Single Image Super-Resolution with Non-Local Balanced Low-Rank Matrix Restoration," IEEE Computer Society, pp. 1255-1260, 2016

7. S. Liu, Z. Pan, and H. Song, "Digital Image Watermarking Method based on DCT and Fractal Encoding," IET Image Processing, Vol. 11, No. 10, pp. 815-821, 2017

8. P. Ferrara and T. Bianchi, "Image Forgery Localization via Fine-Grained Analysis of CFA Artifacts," IEEE Transactions on Information Forensics and Security, Vol. 7, No. 5, pp. 1566-1577, 2012

9. T. F. Long, W. L. Jiao, G. J. He, and W. Wang, "Automatic Line Segment Registration using Gaussian Mixture Model and Expectation-Maximization Algorithm," IEEE Journal of Selected Topics in Applied Earth Observations and Remote Sensing, Vol. 7, No. 5, pp. 1688-1699, 2014

10. S. Liu, "Introduction of Key Problems in Long-Distance Learning and Training," Mobile Networks and Applications, Vol. 24, No. 1, pp. 1-4, 2019

11. G. Gennarelli and F. Soldovieri, "Multipath Ghosts in Radar Imaging: Physical Insight and Mitigation Strategies," IEEE Journal of Selected Topics in Applied Earth Observations and Remote Sensing, Vol. 8, No. 3, pp. 1078-1086, 2014

12. G. Yang and S. Liu, "Distributed Cooperative Algorithm for k-M Set with Negative Integer k by Fractal Symmetrical Property," International Journal of Distributed Sensor Networks, Vol. 10, No. 5, 2014

13. Y. Tu, Y. Lin, J. Wang, and J. U. Kim, "Semi-Supervised Learning with Generative Adversarial Networks on Digital Signal Modulation Classification," CMC-Computers Materials and Continua, Vol. 55, No. 2, pp. 243-254, 2018

14. A. Kumar, R. Pooja, and G. K. Singh, "Design and Performance of Closed form Method for Cosine Modulated Filter Bank using Different Windows Functions,” International Journal of Speech Technology, Vol. 17, No. 4, pp. 427-441, 2014

15. Y. Lin, C. Wang, J. X. Wang, and Z. Dou, "A Novel Dynamic Spectrum Access Framework based on Reinforcement Learning for Cognitive Radio Sensor Networks," Sensors, Vol. 16, No. 10, pp. 1675, 2016

16. M. Liu, S. Liu, W. Fu, and J. T. Zhou, "Distributional Escape Time Algorithm based on Generalized Fractal Sets in Cloud Environment," Chinese Journal of Electronics, Vol. 24, No. 1, pp. 124-127, 2015

17. Q. Y. Tan, H. Leung, Y. Song, et al., "Multipath Ghost Suppression for Through-the-Wall-Radar," IEEE Transactions on Aerospace and Electronic Systems, Vol. 50, No. 3, pp. 2284-2292, 2014

18. S. W. Lyu, X. Y. Pan, and X. Zhang, "Exposing Region Splicing Forgeries with Blind Local Noise Estimation," International Journal of Computer Vision, Vol. 110, No. 2, pp. 202-221, 2014

19. Y. Lin, X. Zhu, Z. Zheng, Z. Dou, and R. L. Zhu, "The Individual Identification Method of Wireless Device based on Dimensionality Reduction and Machine Learning," Journal of Supercomputing, Vol. 75, No. 6, pp. 3010-3027, 2019 